\title{
PRECOMPLETE EQUIVALENCE RELATIONS IN DOMINICAL CATEGORIES
}

\author{
CLAUDIO BERNARDI ${ }^{1}$
}

\begin{abstract}
In this paper precomplete equivalence relations are introduced in dominical and recursion categories. After recalling definitions in $\$ 2$, in $\$ 3$ an analogue of Erకov's Fixed Point Theorem is proved, as well as the recursive inseparability of two disjoint (unions of) equivalence classes. In $\$ 4$ "morphisms" between equivalence relations are discussed, while $\$ 5$ is devoted to the construction of an example (a new characterization of precomplete equivalence relations is also supplied in Lemma 5).
\end{abstract}

1. Introduction. With regard to the general framework for an algebraic treatment of incompleteness phenomena, R. A. Di Paola and A. Heller have recently suggested a categorical approach to recursion theory. This approach is very elegant and compact, since they have succeeded in obtaining many of the main results of classical recursion theory starting from a few appropriate axioms. In this way the very substantial recursive properties are clarified, to which the incompleteness of the usual first order theories corresponds (see [DPH] for more discussion, examples, etc.; see [DP, Ro], and, in particular, [He] for short surveys on some aspects of this subject).

The aim of the present paper is to carry this program further by introducing Eršov's notion of precompleteness in Di Paola and Heller's dominical and recursion categories. In fact in [Vi, BS, BM] precomplete equivalence relations have been shown to be intimately related to incompleteness phenomena; hence this study contributes to the general project. The consideration of precomplete equivalence relations is useful also for another reason. Indeed, it leads to the construction of an example of a recursion category with unexpected properties: for instance every morphism can be extended to a total morphism and every nontrivial domain is creative and complete. This example furnishes additional evidence that many recursive pathologies - such as partial recursive functions with no total extensions, simple sets, incomparable r.e. sets-can be regarded as not inherent in incompleteness phenomena.

Received by the editors December 28, 1984.

1980 Mathematics Subject Classification. Primary 03D75.

${ }^{1}$ Partially supported by a grant from the Italian CNR (U.S.-Italy Cooperative Science Program supported by CNR-NSF). This paper was completed while the author was a visiting professor at Queens College (CUNY). In this regard, much gratitude is expressed to those who have made this invitation possible. 
ACKNOWLEDGMENT. As I have discussed several problems related to the subject of the present paper with Robert A. Di Paola, Alex Heller, Massimo Mirolli, Daniela Monteverdi, Giuseppe Rosolini, Andrea Sorbi, Stefano Stefani, I am indebted to all of them.

2. Basic definitions. Let us briefly recall the following definitions from [DPH]. Let $\mathrm{C}$ be a category in which for every object $X, Y$ there is a morphism $0: X \rightarrow Y$ such that $0 \phi=\phi 0=0$; call a morphism $f$ total if, for every $\phi$, from $f \phi=0$ it follows that $\phi=0$. C is a dominical category if there is a bifunctor (near-product) $\times$ : $\mathbf{C} \times \mathbf{C} \rightarrow \mathbf{C}$ such that: (1) $\phi \times \psi=0$ iff $\phi=0$ or $\psi=0 ;(2) \times$ restricts to the subcategory with only the total morphisms, where it becomes a product, accompanied by projections $p_{i}: X_{1} \times X_{2} \rightarrow X_{i}$ where $i \in\{1,2\}$; (3) the associativity and symmetry isomorphisms of this restriction are natural; (4) for every $\phi: X \rightarrow Y$ and every $Z, p_{1}(\phi \times Z)=\phi p_{1}$ and $(\phi \times \phi) \Delta_{X}=\Delta_{Y} \phi$ where $\Delta_{X}: X \rightarrow X \times X$ is the diagonal morphism. The domain of a morphism $\phi: X \rightarrow Y$ is defined as follows: $\operatorname{dom} \phi=p_{1}(X \times \phi) \Delta_{X}$.

We will adopt notation as in [DPH]. In particular, for any object $X$ the symbol $X$ denotes also the identity morphism, and the symbol $\langle\phi \psi\rangle$ stands for $(\phi \times \psi) \Delta_{X}$. In order to avoid notational complexity we will simply write $\Delta$, omitting the subscript, and will intend that $\operatorname{dom} \phi \psi$ means $\operatorname{dom}(\phi \psi)$. As usual Latin letters denote total morphisms.

Proposition 1 (SEe [DPH AND Ro]). For all morphisms $\phi$ and $\psi$

(i) $\operatorname{dom} \operatorname{dom} \phi=\operatorname{dom} \phi$ (a morphism $\varepsilon$ which equals $\operatorname{dom} \varepsilon$ is said to be a domain);

(ii) $\operatorname{dom} \phi \psi=\operatorname{dom}(\operatorname{dom} \phi) \psi$ and $\phi \operatorname{dom} \psi \phi=(\operatorname{dom} \psi) \phi$;

(iii) $(\operatorname{dom} \phi)(\operatorname{dom} \psi)=\operatorname{dom}\langle\phi \psi\rangle=(\operatorname{dom} \psi)(\operatorname{dom} \phi)$ and $\operatorname{dom} \phi \times \psi=(\operatorname{dom} \phi)$ $\times(\operatorname{dom} \psi)$;

(iv) $\operatorname{dom} \phi \psi \subseteq \operatorname{dom} \psi$ (if $\delta$ and $\varepsilon$ are domains, $\delta \subseteq \varepsilon$ means $\delta \varepsilon=\delta$ );

(v) $\phi=0$ iff $\operatorname{dom} \phi=0$, and $\psi: X \rightarrow Y$ is total iff $\operatorname{dom} \psi=X$.

A Turing morphism is a morphism $\tau: X \times X \rightarrow X$ such that for every $\phi$ : $X \times X \rightarrow X$ there is a total $g: X \rightarrow X$ with $\phi=\tau(g \times X)$; if $\psi: X \rightarrow X$ we say that $g$ is an index for $\psi$ (relative to $\tau$ ) if $\psi p_{2}=\tau(g \times X)$. A dominical category possessing a Turing morphism and in which any two objects are isomorphic is called a recursion category.

Finally, by an equivalence relation (on an object $X$ ) we mean a domain $\eta$ in $X \times X$ such that $\eta \Delta=\Delta, \eta=\operatorname{dom} \eta t$ (where $t$ is the transposition) and $(\eta \times X) \times$ $(X \times \eta) \subseteq \operatorname{dom} \eta p_{1,3}$.

Now let us also recall that in classical recursion theory an equivalence relation $R$ on $\omega$ is said to be precomplete if for every partial recursive function $\psi$ there is a total recursive function $h$ such that, for every $n$, if $\psi$ converges on $n$ then $\psi n R h n$. As an example, let $\simeq$ be defined as follows: $x \simeq y$ iff one can prove (for instance in PA) that $x$ and $y$ are indices for the same partial recursive function: it is not difficult to prove that $\simeq$ is precomplete. Moreover, if $\chi$ is a universal function, define a relation $S_{\chi}$ as follows: $x S_{\chi} y$ iff there exist $n, m$ such that $\chi^{n} x=\chi^{m} y$ (where 
$\chi^{0} x=x$ ). Then $S_{x}$ can be proved to be precomplete. See [Vi, BS] for other examples and general discussions; here we recall only the following

Proposition 2. (a) (Eršov's Fixed Point Theorem) If $R$ is precomplete, then for every total recursive function $f$ there is a number $n$ such that $f n R n$. (If the considered function $\psi$ is not assumed to be total, then there is an $n$ such that if $\psi$ converges on $n$ then $\psi n R n$.)

(b) If $R$ is precomplete, then any two distinct equivalence classes are recursively inseparable (and in fact effectively inseparable).

From Proposition 2(a) and from the fact that the relation $\simeq$ defined before is precomplete, the Recursion Theorem immediately follows.

3. Results on precomplete equivalence relations. Throughout this section, the category with which we are dealing is understood to be a recursion category. Moreover, when speaking of morphisms and equivalence relations, we always mean morphisms from $X$ to $X$ and equivalence relations on $X$, where $X$ is a fixed object.

Definition 1. An equivalence relation $\eta$ in a dominical category is said to be precomplete if for every morphism $\psi$ there exists a total morphism $h$ such that $\operatorname{dom} \eta\langle\psi h\rangle=\operatorname{dom} \psi$.

The given definition appears as the completely faithful translation of the usual one; of course, in some contexts one may prefer to exclude the case $\eta=X \times X$. As in the classical case, we say that $h$ makes $\psi$ total (modulo $\eta$ ). Notice that a relation which contains a precomplete one is in turn precomplete.

REMARK. From a categorical point of view, a precomplete equivalence relation enjoys the following property. Let $c$ be a total morphism such that $c p_{1} \eta=c p_{2} \eta$. Then for every morphism $\psi$ there is a total morphism $h$ such that $\operatorname{dom} \psi$ is an equalizer of $c \psi$ and $c h$. Indeed, we have

$$
c \psi \operatorname{dom} \psi=c \psi=c p_{1} \Delta \psi=c p_{1} \eta \Delta \psi=c p_{1} \eta(\psi \times h) \Delta=c p_{2} \eta(\psi \times h) \Delta ;
$$

and similarly

$$
c h \operatorname{dom} \psi=c p_{2} \eta \Delta h \operatorname{dom} \psi=c p_{2} \eta(h \operatorname{dom} \psi \times h) \Delta \operatorname{dom} \psi=c p_{2} \eta(\psi \times h) \Delta \operatorname{dom} \psi \text {. }
$$

By precompleteness we can deduce $c \psi \operatorname{dom} \psi=c h \operatorname{dom} \psi$. On the other hand, assume $c \psi \phi=c h \phi$ : we claim that $\phi$ itself is the only morphism $\chi$ (whose domain is contained in $\operatorname{dom} \phi)$ such that $\phi=(\operatorname{dom} \psi) \chi$. Actually, since $\operatorname{dom} \psi \phi=\operatorname{dom} \phi$, we have $(\operatorname{dom} \psi) \phi=\phi \operatorname{dom} \psi \phi=\phi \operatorname{dom} \phi=\phi$.

THEOREM 1. If $\eta$ is a precomplete equivalence relation, then for any morphism $\phi$ there exists a total morphism $g$ such that $\operatorname{dom} \eta\langle\phi X\rangle g=\operatorname{dom} \phi g$.

Proof. Let $h$ be a morphism which makes $\phi \tau \Delta$ total and let $w$ be an index of $h$ so that $\tau(w \times X)=h p_{2}$. We claim that the total morphism $g=h w$ satisfies the condition in the statement. First of all, note that

$$
\tau \Delta w=\tau(w \times X)(X \times w) \Delta=h p_{2}(X \times w) \Delta=h w p_{2} \Delta=h w .
$$

Now we have

$$
\begin{aligned}
\eta\langle\phi X\rangle \tau \Delta w & =\eta(\phi \times X)(\tau \Delta \times \tau \Delta w)(w \times X) \Delta \\
& =\eta(\phi \tau \Delta \times h w)(w \times X) \Delta=\eta\langle\phi \tau \Delta h\rangle w .
\end{aligned}
$$


By the definition of $h, \operatorname{dom} \eta\langle\phi \tau \Delta h\rangle=\operatorname{dom} \phi \tau \Delta$; therefore

$$
\begin{aligned}
\operatorname{dom} \eta\langle\phi X\rangle h w & =\operatorname{dom} \eta\langle\phi X\rangle \tau \Delta w=\operatorname{dom} \eta\langle\phi \tau \Delta h\rangle w=\operatorname{dom}(\operatorname{dom}\langle\phi \tau \Delta h\rangle) w \\
& =\operatorname{dom}(\operatorname{dom} \phi \tau \Delta) w=\operatorname{dom} \phi \tau \Delta w=\operatorname{dom} \phi h w .
\end{aligned}
$$

REMARK. The preceding result can be expressed by saying that for every $\phi$ there exists a total morphism $g$ which makes $\phi g$ total.

COROllaRY 1 (FIXED PoINT THEOREM). If $\eta$ is a precomplete equivalence relation, then every total morphism $f$ admits a fixed point $g$, in the sense that $\eta\langle f g g\rangle$ is total (and hence $\eta\langle f X\rangle \neq 0$ ).

Proof. Substitute $f$ for $\phi$ in Theorem 1 and note that dom $f g=X$.

COROLLARY 2. Let $\gamma$ be a diagonal morphism for a precomplete equivalence relation $\eta$, in the sense that $\eta\langle\gamma X\rangle=0$. Then $\gamma$ cannot be total.

To obtain the Recursion Theorem as a direct consequence of the Fixed Point Theorem, we need an analogue of the relation $\simeq$ (recall that $x \simeq y$ iff $x$ and $y$ are provably indices of the same partial recursive function). First, note that it immediately follows from the definition of a Turing morphism that for every morphism $\psi$ there exists a total morphism $w$ such that $\tau(\psi \times X)=\tau(w \times X)$. Now, if we assume that there exists an equivalence relation $\eta$ such that (1) for any $\psi$ and $w$ as before the equality $\operatorname{dom} \eta\langle\psi w\rangle=\operatorname{dom} \psi$ holds, and (2) if $\eta\langle f g\rangle$ is total then $\tau(f \times X)=\tau(g \times X)$, then we can easily deduce the Recursion Theorem. Indeed, by condition (1) $\eta$ is precomplete; therefore, by applying the Fixed Point Theorem we get that for every total morphism $f$ there exists a total morphism $g$ such that $\eta\langle f g g\rangle$ is total. By condition (2) we can conclude $\tau(f g \times X)=\tau(g \times X)$.

Regarding equivalence relations generated by universal functions, we start by giving

DEFINITION 2. A morphism $\chi$ in a dominical category is said to be universal if for every morphism $\psi$ there exists a total morphism $g$ such that $\psi=\chi g$.

EXAMPLE. If $\tau$ is a Turing morphism, then $\tau \Delta$ is a universal morphism. Indeed, given $\psi$, let $g$ be such that $\tau(g \times X)=\psi p_{1}$; in view of Lemma 3.2 of [DPH], we have

$$
\tau \Delta g=\tau(g \times X)(X \times g) \Delta=\psi p_{1}(X \times g) \Delta=\psi p_{1}(X \times \operatorname{dom} g) \Delta=\psi .
$$

One can readily prove that if $h$ makes $\chi$ total modulo $\eta$, then, for every total morphism $g, h g$ makes $\chi g$ total modulo $\eta$ : it follows that a relation $\eta$ is precomplete iff a universal morphism can be made total modulo $\eta$.

Simply translating the concept of a relation $\eta$ (containing the one) generated by a morphism $\chi$, we get the following condition: $\operatorname{dom} \eta\langle\chi \quad X\rangle=\operatorname{dom} \chi$. From this point of view it is quite obvious that if $\chi$ is universal then $\eta$ is precomplete. (Keeping the previous fact in mind, it is enough to notice that the identity makes $\chi$ total modulo $\eta$.) Actually, a similar argument applies in the more general case in which $\chi$ is universal modulo $\eta$, in the sense that for every $\psi$ there exists a $g$ such that $\operatorname{dom} \eta\langle\psi \chi g\rangle=\operatorname{dom} \psi$ (transitivity of $\eta$ is used). 
REMARK. It directly follows from the definition that the domain $\delta$ of a universal morphism is complete, according to Definition 8.8 of [DPH]; as a consequence of a recent result by $\mathrm{A}$. Heller, $\delta$ is also creative.

We conclude this section by showing that, if additional hypotheses are assumed about the considered dominical category, other properties of precomplete equivalence relations can be proved. The following theorem extends the property expressed by Proposition 2(b) to suitable dominical categories. First we state a simple technical lemma.

LEMMA 1. If $\delta$ is a domain, from $\operatorname{dom} \phi g=\operatorname{dom} \psi g$ it follows that $\operatorname{dom} \phi \delta g=$ $\operatorname{dom} \psi \delta g$.

Proof. By Proposition 1, from the given equality we can deduce successively $g \operatorname{dom} \phi g=g \operatorname{dom} \psi g, \quad(\operatorname{dom} \phi) g=(\operatorname{dom} \psi) g, \quad(\operatorname{dom} \phi) \delta g=(\operatorname{dom} \psi) \delta g$, $\operatorname{dom}(\operatorname{dom} \phi) \delta g=\operatorname{dom}(\operatorname{dom} \psi) \delta g, \operatorname{dom} \phi \delta g=\operatorname{dom} \psi \delta g$.

Definition 3. A domain $\varepsilon$ is a union of equivalence classes with respect to an equivalence relation $\eta$ if, for every domain $\delta$, from $\varepsilon \delta=0$ it follows that $\eta(\varepsilon \times \delta)$ $=0 .{ }^{2}$

THEOREM 2. Let $\varepsilon_{1}$ and $\varepsilon_{2}$ be two unions of equivalence classes with respect to a precomplete equivalence relation $\eta$ in a ct-dominical category (see [DPH] for the definition). If $\varepsilon_{1}$ and $\varepsilon_{2}$ are nonempty and $\varepsilon_{1} \varepsilon_{2}=0$, then $\varepsilon_{1}$ and $\varepsilon_{2}$ are recursively inseparable.

Proof. Suppose to the contrary that there is a complemented domain $\delta$ such that $\varepsilon_{1} \subseteq \delta$ and $\varepsilon_{2} \subseteq \delta^{\perp}$. Since the category is $c+$-dominical, by Proposition 6.6 of [DPH] there are total $f_{1}$ and $f_{2}$ such that $\varepsilon_{1} f_{1}=f_{1}$ and $\varepsilon_{2}=f_{2}=f_{2}$. Moreover, by Proposition 7.6 of [DPH] there exists a morphism $\psi$ such that $\psi \delta=f_{2} \delta$ and $\psi \delta^{\perp}=f_{1} \delta^{\perp}$ (note that $\operatorname{dom} f_{2} \delta=\delta$ and $\operatorname{dom} f_{1} \delta^{\perp}=\delta^{\perp}$ ).

Now, by applying Theorem 1 we get a total morphism $g$ such that

$$
\operatorname{dom} \eta\langle\psi X\rangle g=\operatorname{dom} \psi g \text {; }
$$

by Lemma 1 we can also write $\operatorname{dom} \eta\langle\psi X\rangle \delta g=\operatorname{dom} \psi \delta g$. We claim that $\delta g=$ $\delta^{\perp} g=0$, contradicting the fact that $\delta^{\perp}$ is the complement of $\delta$.

Indeed, in view of the fact that $\varepsilon_{2} \subseteq \delta^{\perp}$ and $\varepsilon_{2}$ is a union of equivalence classes, $\eta\left(\varepsilon_{2} \times \delta\right)=0$. We can deduce successively that

$$
\begin{aligned}
0 & =\eta\left(\varepsilon_{2} \times \delta\right)\left(f_{2} \times X\right) \Delta \delta g=\eta\left\langle\varepsilon_{2} f_{2} \delta\right\rangle \delta g=\eta\left\langle f_{2} \delta\right\rangle \delta g \\
& =\eta\left\langle f_{2} \delta \delta\right\rangle g=\eta\langle\psi \delta \delta\rangle g=\eta\langle\psi X\rangle \delta g .
\end{aligned}
$$

Therefore

$$
0=\operatorname{dom} \eta\langle\psi X\rangle \delta g=\operatorname{dom} \psi \delta g=\operatorname{dom} f_{2} \delta g=\operatorname{dom}\left(\operatorname{dom} f_{2}\right) \delta g=\operatorname{dom} \delta g ;
$$

and we conclude that $\delta g=0$. Similarly we can prove $\delta^{\perp} g=0$.

4. Morphisms between equivalence relations. The introduction of morphisms between equivalence relations in dominical categories is suggested by two reasons. $\mathrm{A}$

\footnotetext{
${ }^{2} \mathrm{~A}$ slightly stronger condition is $\eta(\varepsilon \times X) \subseteq \eta(\varepsilon \times \varepsilon)$. In an $r$-dominical category this condition is equivalent to $\varepsilon=$ range $p_{2} \eta(\varepsilon \times X)$, that is, to the fact that $\varepsilon$ is $\eta$-saturated.
} 
first objective is the translation into this more general context of the classical results which, as the ones proved in [BS, BM], rest on the possibility of comparing equivalence relations. Second, we make available a general procedure for constructing new examples of dominical categories (we will see an application of this procedure in the following section).

Let us start by

Definition 4. Let $\psi: X \rightarrow Y$ be a morphism and let $\eta, \theta$ be two equivalence relations on $X, Y$ respectively. We say that $\psi$ is an $\eta-\theta$ morphism and we write $\psi$ : $(X, \eta) \rightarrow(Y, \theta)$ if $\eta((\operatorname{dom} \psi) \times X) \subseteq \operatorname{dom} \theta(\psi \times \psi) .^{3}$

LEMMA 2. $X$ is a morphism from $(X, \eta)$ to $(X, \theta)$ iff $\eta \subseteq \theta$. Given two morphisms $\phi:(X, \eta) \rightarrow(Y, \theta)$ and $\psi:(Y, \theta) \rightarrow(Z, \zeta)$, the composition $\psi \phi$ is an $\eta-\zeta$ morphism. A total morphism $h$ is an $\eta-\theta$ morphism iff $(h \times h) \eta \subseteq \theta(h \times h)$.

Proof. We omit the proof. (The second and third statements are slightly less obvious than one may expect. However, they follow from Proposition 1.)

Given a dominical category $\mathbf{C}$, we can define a new dominical category $\overline{\mathbf{C}}$ as follows. As objects we consider the pairs $(X, \eta)$ where $\eta$ is an equivalence relation on $X$. The near product is defined as follows:

$$
(X, \eta) \times(Y, \theta)=(X \times Y,(X \times t \times Y)(\eta \times \theta)(X \times t \times Y)) .
$$

To define the morphisms, first we give

Definition 5. Two morphisms $\phi, \psi:(X, \eta) \rightarrow(Y, \theta)$ are said to be equivalent if $\operatorname{dom} \theta\langle\phi \psi\rangle=\operatorname{dom} \phi=\operatorname{dom} \psi$. We will write $\phi \sim \psi$.

LEMMA 3. (a) is an equivalence relation in the set of $\eta-\theta$ morphisms.

(b) is a congruence, that is:

(b.1) if $\phi_{1}, \psi_{1}:(X, \eta) \rightarrow(Y, \theta)$ are equivalent and $\phi_{2}, \psi_{2}:(Y, \theta) \rightarrow(Z, \zeta)$ are equivalent, then $\phi_{2} \phi_{1} \sim \psi_{2} \psi_{1}$, and

(b.2) if $\phi_{i}, \psi_{i}:\left(X_{i}, \eta_{i}\right) \rightarrow\left(Y_{i}, \theta_{i}\right)$ are equivalent for $i \in\{1,2\}$, then $\phi_{1} \times \phi_{2} \sim \psi_{1}$ $\times \psi_{2}$.

Proof. (a) We only sketch the proof of transitivity. If $\phi \sim \psi$ and $\psi \sim \chi$, then one can prove that $\operatorname{dom}(\theta \times Y)(Y \times \theta)(\phi \times \psi \times \chi)(X \times \Delta) \Delta=\operatorname{dom} \phi$. On the other hand

$$
\begin{aligned}
\operatorname{dom}\left(\operatorname{dom} \theta p_{1,3}\right)(\phi \times \psi \times \chi)(X \times \Delta) \Delta & \subseteq \operatorname{dom} \theta p_{1,3}(\phi \times X \times \chi)(X \times \Delta) \Delta \\
& =\operatorname{dom} \theta(\phi \times \chi) \Delta .
\end{aligned}
$$

By the transitivity of $\theta$ we conclude that $\operatorname{dom} \phi \subseteq \operatorname{dom} \theta\langle\phi \chi\rangle$. The proofs of the other inclusions are similar or immediate.

(b.1) We omit the tedious proof which depends on (a) and Proposition 1.

(b.2) We have to prove that

$$
A=\operatorname{dom}\left(Y_{1} \times t \times Y_{2}\right)\left(\theta_{1} \times \theta_{2}\right)\left(Y_{1} \times t \times Y_{2}\right)\left(\phi_{1} \times \phi_{2} \times \psi_{1} \times \psi_{2}\right) \Delta_{X_{1} \times X_{2}}
$$

equals

$$
\operatorname{dom} \phi_{1} \times \phi_{2}=\left(\operatorname{dom} \phi_{1}\right) \times\left(\operatorname{dom} \phi_{2}\right)=\operatorname{dom} \psi_{1} \times \psi_{2}
$$

\footnotetext{
${ }^{3}$ Similarly, if $\psi: X \times Y \rightarrow Z$ and $\eta, \theta, \zeta$ are equivalence relations on $X, Y, Z$ respectively, $\psi$ is an $\eta \times \theta-\zeta$ morphism if $(\eta \times \theta)(X \times t \times Y)((\operatorname{dom} \psi) \times X \times Y) \subseteq \operatorname{dom} \zeta(\psi \times \psi)$.
} 
In fact we have

$$
\begin{aligned}
A & =\operatorname{dom}\left(\theta_{1} \times \theta_{2}\right)\left(Y_{1} \times t \times Y_{2}\right)\left(\phi_{1} \times \phi_{2} \times \psi_{1} \times \psi_{2}\right)\left(X_{1} \times t \times X_{2}\right)\left(\Delta_{X_{1}} \times \Delta_{X_{2}}\right) \\
& =\operatorname{dom}\left(\theta_{1} \times \theta_{2}\right)\left(\phi_{1} \times \psi_{1} \times \phi_{2} \times \psi_{2}\right)\left(\Delta_{X_{1}} \times \Delta_{X_{2}}\right) \\
& =\left(\operatorname{dom} \theta_{1}\left(\phi_{1} \times \psi_{1}\right) \Delta_{X_{1}}\right) \times\left(\operatorname{dom} \theta_{2}\left(\phi_{2} \times \psi_{2}\right) \Delta_{X_{2}}\right) \\
& =\left(\operatorname{dom} \phi_{1}\right) \times\left(\operatorname{dom} \phi_{2}\right) .
\end{aligned}
$$

The preceding lemma allows us to assume the equivalence classes of $\eta-\theta$ morphisms from $X$ to $Y$ as morphisms from $(X, \eta)$ to $(Y, \theta)$ in $\overline{\mathbf{C}}$. In this way, $\overline{\mathbf{C}}$ is in turn a dominical category.

LeMma 4. If two $\eta-\theta$ morphisms $\phi, \psi$ are equivalent, then $\operatorname{dom} \theta(\phi \times \psi)=$ $\operatorname{dom} \theta(\phi \times \phi)$.

Proof. The proof is by Lemma 3.

Henceforth, for the sake of simplicity, we will consider only total $\eta-\theta$ morphisms. In view of the following results, this definition seems to be the most appropriate one.

Definition 6. A morphism $h:(X, \eta) \rightarrow(Y, \theta)$ is said to be (a) injective if $\eta=\operatorname{dom} \theta(h \times h)$; (b) surjective if there exists a $k:(Y, \theta) \rightarrow(X, \eta)$ such that $h k \sim Y$; (c) bijective if there exists a $k:(Y, \theta) \rightarrow(X, \eta)$ such that $h k \sim Y$ and $k h \sim X$.

REMARK. In other words, an $\eta-\theta$ morphism $h$ in $\mathbf{C}$ is surjective iff the corresponding morphism in $\overline{\mathbf{C}}$ is a split epimorphism. On the other hand, if the morphism corresponding to $h$ in $\overline{\mathbf{C}}$ is a split monomorphism, then $h$ is injective according to Definition 6. Indeed, assume that there exists a $k:(Y, \theta) \rightarrow(X, \eta)$ such that $k h \sim X$. We have

$$
\operatorname{dom} \theta(h \times h) \subseteq \operatorname{dom}(\operatorname{dom} \eta(k \times k))(h \times h)=\operatorname{dom} \eta(k h \times X)(X \times k h)
$$

which by Lemma 4 equals $\eta$.

TheOREM 3. (a) A morphism $h:(X, \eta) \rightarrow(Y, \theta)$ is bijective iff it is both injective and surjective.

(b) If $h$ is injective, from $h f \sim$ hg it follows that $f \sim g$ (with $f, g \zeta-\eta$ morphisms).

(c) If $h$ is surjective, from $f h \sim$ gh it follows that $f \sim g$ (with $f, g \theta-\zeta$ morphisms).

Proof. (a) One direction follows from the previous remark. Conversely, we have

$$
\begin{aligned}
\operatorname{dom} \eta\langle k h X\rangle & =\operatorname{dom}(\operatorname{dom} \theta(h \times h))(k h \times X) \Delta=\operatorname{dom} \theta(h k \times Y) \Delta h \\
& =\operatorname{dom} Y h=X .
\end{aligned}
$$

(b) This proof is straightforward.

(c) Consider $f h k$ and $g h k$ and apply Lemma 3.

We conclude with two theorems concerning surjective morphisms. These theorems are the translations to dominical categories of results proved in classical recursion theory in [BM]. (The second one, in which precompleteness is involved, is proved also in [LM]; as stated there, it needs a further hypothesis, since non-r.e.-equivalence relations are considered as well.) 
TheOREM 4. A morphism $h:(X, \eta) \rightarrow(Y, \theta)$ is surjective iff there exist an equivalence relation $\zeta$ (on $X$ ) containing $\eta$ (in the sense that $\zeta \eta=\eta$ ) and a bijective morphism $f:(X, \zeta) \rightarrow(Y, \theta)$.

Proof. $(\Rightarrow)$ Since $h$ is surjective, there exists a $k:(Y, \theta) \rightarrow(X, \eta)$ according to Definition 6. Now define $\zeta$ to be $\operatorname{dom} \theta(h \times h)$. Note that $h$ is obviously a $\zeta-\theta$ morphism, as well as $k$ is a $\theta-\zeta$ morphism. Indeed,

$$
\operatorname{dom} \zeta(k \times k)=\operatorname{dom} \theta(h k \times h k)=\theta
$$

by Lemma 4 . Finally, to show that $h$ is bijective, we only have to prove that $\zeta(k h \times X) \Delta$ is total, the other condition being granted by the definition of $k$. We have

$$
\operatorname{dom} \zeta(k h \times X) \Delta=\operatorname{dom} \theta(h k h \times h) \Delta=\operatorname{dom} \theta(h k \times Y) \Delta h=\operatorname{dom} \theta \Delta h=X
$$

(we have applied Lemma 4 and the reflexivity of $\theta$ ).

$(\Leftarrow) f$ itself is a surjective $\eta-\theta$ morphism. We omit the easy proof.

THEOREM 5. A surjective morphism preserves precompleteness, in the sense that if $h$ : $(X, \eta) \rightarrow(X, \theta)$ is surjective and $\eta$ is precomplete, then $\theta$ is in turn precomplete.

Proof. Given any morphism $\psi$, let $f$ be the morphism which makes $k \psi$ total modulo $\eta$, where $k$ is as in Definition 6. By applying Lemma 4 we can write successively

$$
\begin{aligned}
\operatorname{dom} \psi & =\operatorname{dom}(\operatorname{dom} k) \psi=\operatorname{dom} k \psi=\operatorname{dom} \eta\langle k \psi f\rangle \\
& \subseteq \operatorname{dom} \theta(h \times h)\langle k \psi f\rangle=\operatorname{dom} \theta(h k \times X)\langle\psi h f\rangle=\operatorname{dom} \theta\langle\psi h f\rangle .
\end{aligned}
$$

On the other hand, $\operatorname{dom} \theta\langle\psi h f\rangle \subseteq \operatorname{dom}\langle\psi X\rangle=\operatorname{dom} \psi$. Thus we can conclude that $h f$ makes $\psi$ total modulo $\theta$.

5. An example of a recursion category. Now, referring to a precomplete equivalence relation and to the concepts introduced in the previous section, we construct a recursion category $\mathbf{D}$ which has unusual properties. In a sense, in this category incompleteness phenomena are more frequent, yet more uniform.

Given any r.e. equivalence relation $\eta_{1}$ (in the classical meaning), we can associate with it the r.e. equivalence relation $\eta_{2}$ defined as follows: $x \eta_{2} y$ iff one can prove (for instance in PA) that, for every $n, \phi_{x}$ converges on $n$ iff $\phi_{y}$ does and, if this is the case, $\phi_{x} n \eta_{1} \phi_{y} n$.

Let $\eta$ be a fixed point of the operator which maps every $\eta_{1}$ in the corresponding $\eta_{2}$ (the existence of such a fixed point is assured by the Recursion Theorem and by the possibility of an effective enumeration of r.e. equivalence relations; see [BS]). Note that $\eta$ cannot be $\omega \times \omega$ (since $x \eta y$ implies $W_{x}=W_{y}$ ) and is precomplete because it contains the precomplete equivalence relation $\simeq$ defined in $\S 2$.

The desired category $\mathbf{D}$ is obtained considering the classical recursion category $\mathbf{T}$ and following a procedure very similar to the one which leads to the construction of the category $\overline{\mathbf{C}}$ (see $\$ 4$ ). Recall that the set of the objects of $\mathbf{T}$ is constructed starting from a set $H$ of denumerable sets and closing $H$ under pairwise products. On each set $X$ in $H$ introduce an equivalence relation $\eta_{X}$ isomorphic to $\eta$; in the product 
$X \times Y$ introduce the relation $\eta_{X} \times \eta_{Y}$. We can define a bijection $h$ from the Cartesian square of $\omega / \eta$ to $\omega / \eta$ as follows:

$$
h\left([x]_{\eta},[y]_{\eta}\right)=[z]_{\eta} \quad \text { where } \phi_{z} n= \begin{cases}\phi_{x}(n / 2) & \text { if } n \text { is even, } \\ \phi_{y}(n-1) / 2 & \text { if } n \text { is odd }\end{cases}
$$

Therefore all the relations introduced in the objects of $\mathbf{T}$ are isomorphic. We denote all of them by the same symbol $\eta$.

The objects of $\mathbf{D}$ are all the pairs of the form $(X, \eta)$. Then consider the set $M$ of partial recursive functions which can be proved to be $\eta-\eta$ morphisms and the equivalence relation $\sim$ in $M$ : the morphisms of $\mathbf{D}$ are the $\sim$ equivalence classes. This makes $\mathbf{D}$ a dominical category. To show that $\mathbf{D}$ is a recursion category, we construct a Turing morphism. Let $\tau$ be a Turing morphism in the classical recursion category $\mathbf{T}$. Define $\tau_{\eta}$ as follows:

$$
\tau_{\eta}(x, y)= \begin{cases}\tau(x, y) & \text { if } x \text { is an index for a function in } M, \\ \text { divergent } & \text { otherwise. }\end{cases}
$$

Now, on the one hand $\tau_{\eta} \in M$ (we omit the straightforward proof); on the other, $\left[\tau_{\eta}\right]_{-}$is a Turing morphism for $\mathbf{D}$. To prove this, given a $\psi:(X, \eta) \times(X, \eta) \rightarrow(X, \eta)$ in $M$, consider a total recursive function $g$ such that $\psi(x, y)$ is provably equal to $\phi_{g(x)} y$. We claim that $g \in M$ and hence we can write $[\psi]_{\sim}=\left[\tau_{\eta}\right]_{\sim}\left([g]_{\sim} \times X\right)$. Indeed, assume $x_{1} \eta x_{2}$. Since $\psi \in M$ we can prove that, for every $y, \psi\left(x_{1}, y\right) \eta$ $\psi\left(x_{2}, y\right)$ (if they converge), i.e. $\phi_{g\left(x_{1}\right)} y \eta \phi_{g\left(x_{2}\right)} y$. By the definition of $\eta$, this implies $g\left(x_{1}\right) \eta g\left(x_{2}\right)$.

The following theorems state some significant properties of $\mathbf{D}$.

THEOREM 6. Every morphism $\phi$ in $\mathbf{D}$ can be extended to a total morphism $f$ (in the sense that $f \operatorname{dom} \phi=\phi)$. Therefore every equivalence relation on an object of $\mathbf{D}$ is precomplete.

PROOF. The proof is a direct consequence of

LEMMA 5. Let $\eta$ be a precomplete equivalence relation (on $\omega$ ) and let $\theta$ be any equivalence relation. Any $\theta-\eta$ morphism $\psi$ can be made total by a $\theta-\eta$ morphism $h$.

Proof. We omit the rather technical construction of the function $h$ (one proceeds by induction). Actually the construction is similar to the one of Theorem 3 in [BM], even if at first glance the statement there seems to be completely different. Theorem 3 of $[\mathbf{B M}]$ is in turn a corollary of Lemma 5.

REMARK. The property stated in Lemma 5 obviously implies that $\eta$ is precomplete (assume $\theta$ to be the diagonal relation just to get the usual definition). On the other hand, the fact that any $\eta-\eta$ morphism can be made total by an $\eta-\eta$ morphism does not imply that $\eta$ is precomplete (as an easy counterexample, consider the equivalence relation which associates two numbers $x, y$ iff $x+y$ is even).

THEOREM 7. In $\mathbf{D}$ there are sufficiently many atoms, and each of them is a constant (see [DPH] for definitions).

Proof. Of course, every morphism which maps $\omega$ into a single equivalence class is an atom. 
THEOREM 8. Every domain $\varepsilon$, different from both 0 and $(X, \eta)$, is complete. Therefore there are nonisomorphic complete domains.

Proof. Suppose first that $\varepsilon$ is an atom. Let a domain $\delta$ be given (for the sake of simplicity we consider domains as r.e. subsets of $\omega)$. Define $\eta^{\delta}=\eta \cup(\delta \times \delta)$. Now recall that, given two equivalence relations $R, S, R \leqslant_{\mathrm{m}} S$ means that there is an injective $R-S$ morphism. In [BS] precomplete equivalence relations have been shown to be complete with respect to this reducibility. Therefore $\eta^{\delta} \leqslant_{m} \eta$ and, more precisely, there exists a total recursive function $h$ which is provably an $\eta-\eta$ morphism and such that $h(\delta) \subseteq \varepsilon$. Since $h$ is an injective morphism, $h(\bar{\delta}) \subseteq \bar{\varepsilon}$.

In the general case, consider the (precomplete) relation $\eta^{\varepsilon}=\eta \cup(\varepsilon \times \varepsilon)$ in place of $\eta$. The argument is similar to the previous one. For $h$ to be an $\eta-\eta$ morphism, a slight modification in the construction of Theorem 7 in [BS] is required: namely, assuming $0 \in \delta$ and $a \in \varepsilon$, define $h 0=a$ and then consider $\varepsilon$ in place of $[a]_{\eta}$.

The last statement follows from the first one and Theorem 7.

THEOREM 9. Every domain $\varepsilon$, different from both 0 and $(X, \eta)$, is creative (with respect to indices - see [DPH]). As a consequence, two disjoint nonempty domains are recursively inseparable.

Proof. Recall that dom $\tau \Delta$ is creative and a productive function for it is the identity [DPH, Theorem 8.5]. By Theorem 8 there is a morphism $h$ such that $\operatorname{dom} \varepsilon h=\operatorname{dom} \tau \Delta$. Now, thinking of domains as identity functions defined on r.e. subsets of $\omega$, let a domain $\delta=\phi_{x}$ be given. Obviously dom $\phi_{x} h=\phi_{k x}$. If we prove that $k$ can be chosen to be an $\eta-\eta$ morphism, we can conclude that $h k$ is a productive function for $\varepsilon$. Indeed, assuming that $x \eta y$, we can prove $W_{x}=W_{y}$ and $\phi_{k x}=\phi_{k y} ;$ hence $k x \eta k y$.

THEOREM 10. D is an r-dominical category, but it is neither +-dominical nor c-dominical.

Proof. The first two statements are immediate. To prove the third, we have to find an $\eta-\eta$ morphism such that no section (see [DPH]) of it in T is in turn an $\eta-\eta$ morphism.

Consider the two equivalence relations $\equiv$ and $\nabla$ defined as follows: $x \equiv y$ iff (identifying formulas of PA with their Gödel numbers) $x \leftrightarrow y$ is a theorem, and $x \nabla y$ iff either $x \equiv y$ or $x \equiv \neg y$. By the completeness of precomplete equivalence relations (see [BS]), there exist an injective $\equiv-\eta$ morphism $f$ and an injective $\nabla-\eta$ morphism $h$. Obviously, in $\mathbf{T}$ any injective $\theta-\zeta$ total morphism admits a section which is a surjective $\zeta-\theta$ morphism. Let $g$ be a section of $f$ which is a surjective $\eta-\equiv$ morphism. Since $\equiv \subseteq \nabla$, by Lemma $2 h X g$ is an $\eta-\eta$ morphism. We claim that in D there is no section of $[h X g]$.

Assume to the contrary that $[\sigma]_{\sim}$ is such a section, so that $h X g \sigma h X g \sim h X g$. Since $h$ is injective and $g$ is surjective, by Theorem 3 we have $X g \sigma h X \sim X$ (as $\equiv-\nabla$ morphisms). It follows that the $\nabla-\equiv$ morphism $g \sigma h$ "selects" for every union of the form $[p]_{\equiv} \cup[\neg p]_{\equiv}$ one of the two sets $[p]_{\equiv}$ and $[\neg p]_{\equiv}$. But this is impossible, because, called $A$ the range of $g \sigma h$, the r.e. sets $\{x \mid y \in A$ such that 
$x \equiv y\}$ and $\{x \mid \exists y \in A$ such that $x \equiv \neg y\}$ would separate the sets of theorems and of refutable formulas of PA.

Corollary 3. D is not equivalent to T.

Proof. The proof is obvious since $\mathbf{T}$ is both + -dominical and $c$-dominical.

\section{REFERENCES}

[BM] C. Bernardi and F. Montagna, Equivalence relations induced by extensional formulae: classification by means of a new fixed point property, Fund. Math. 124 (1984), 221-233.

[BS] C. Bernardi and A. Sorbi, Classifying positive equivalence relations, J. Symbolic Logic 48 (1983), 529-538.

[DP] R. A. Di Paola, Creativity and effective inseparability in dominical categories, Atti degli Incontri di Logica Matematica, Vol. 2, Scuola di Specializzazione in Logica Matematica di Siena, Siena, 1985.

[DPH] R. A. Di Paola and A. Heller, Dominical categories, J. Symbolic Logic (to appear).

[He] A. Heller, Dominical categories and recursion theory, Atti degli Incontri di Logica Matematica, Vol. 2, Scuola di Specializzazione in Logica Matematica di Siena, Siena, 1985.

[LM] G. Longo and E. Moggi, The hereditary partial effective functionals and recursion theory in higher types, J. Symbolic Logic 49 (1984), 1319-1332.

[Ro] G. Rosolini, Domini e categorie dominicali, Atti degli Incontri di Logica Matematica, Vol. 2, Scuola di Specializzazione in Logica Matematica di Siena, Siena, 1985.

[Vi] A. Visser, Numerations $\lambda$-calculus and arithmetic, To H. B. Curry: Essays on combinatory logic, lambda calculus and formalism (J. P. Seldin and J. R. Hindley, eds.), Academic Press, London, 1980.

Dipartimento di Matematica, Universita di Siena, Siena, Italy 